\title{
Hormigón estructural de baja densidad para edificaciones
}

\author{
Morales Gubio L. W.*,**; Santamaría Carrera J. L.*,**; Caicedo Barona W.*,*; Tipán Quinatoa F.*,** \\ *Universidad Central del Ecuador, Facultad de Ingeniería, Ciencias \\ Físicas y Matemática, Carrera de Ingeniería Civil, Quito, Ecuador \\ ${ }^{*}$ Autores correspondientes \\ e-mail: 1wmorales@uce.edu.ec \\ Iinformación del artículo \\ Recibido: julio 2018 \\ Aceptado: septiembre 2018
}

\section{Resumen}

El Ecuador se encuentra en una zona de alta peligrosidad sísmica, por lo que el diseño que demanda en las edificaciones de hormigón armado bajo una condición crítica, es el Sismo y siendo la cortante basal, la fuerza lateral aplicada en la edificación proporcional al peso de la estructura, por lo que una alternativa para atenuar esta acción sísmica sobre las estructuras de hormigón armado es disminuir el peso de los elementos estructurales mediante el uso de hormigones ligeros. Por tanto, el objetivo principal de la investigación es fabricar un hormigón de baja densidad que a su vez tenga características estructurales y además se pueda determinar mediante ensayos y correlaciones los parámetros físicos y mecánicos del hormigón ligero para que puedan ser usados como datos dentro del análisis y diseño estructural de edificaciones.

Con base en lo descrito, la investigación consiste en la elaboración de un hormigón estructural de baja densidad con el uso de agregado ligero (piedra pómez) extraída del tramo de vía San Antonio - Calacalí, agregado fino de la cantera Pifo y cemento tipo GU de uso general. El diseño de hormigón ligero estructural se basa en tres normativas: ACI 318-14: Requisitos del Código para Hormigón estructural, ACI 211.1-98: Práctica estándar para seleccionar proporciones de hormigón estructural ligero y ACI 213R-14: Guía para concreto estructural agregado ligero, las cuales proveen de tablas para la estimación de las cantidades de material, dependiendo de la resistencia que se desee alcanzar.

La dosificación para un metro cúbico de hormigón ligero estructural es de 9.2 sacos de cemento, 208 litros de agua, $792 \mathrm{~kg}$ de arena, $326 \mathrm{~kg}$ de piedra pómez y $4.6 \mathrm{~kg}$ de aditivo superplastificante Aditec SF - 106. Utilizando esta dosificación se consigue un hormigón ligero estructural con una resistencia a la compresión a los 28 días de $282 \mathrm{~kg} / \mathrm{cm} 2$ y densidad de equilibrio de 1.81 $\mathrm{kg} / \mathrm{cm} 3$, además, para poder ser usado posteriormente en un modelo matemático, se obtiene el módulo de rotura, resistencia a la tracción, módulo de elasticidad, módulo de Poisson mediante ensayos y fórmulas de correlación del ACI. El hormigón ligero puede ser utilizado en elementos estructurales como vigas, columnas y losas, pero no puede estar con contacto directo con el agua debido a su porosidad. La disminución de la densidad del hormigón trae múltiples ventajas como la reducción de cargas, secciones, cuantía de acero e incluso costo.

Palabras clave: hormigón estructural ligero, piedra pómez, hormigón de baja densidad.

\begin{abstract}
Ecuador is located in an area of high seismic hazard, so the design demanded in reinforced concrete buildings under a critical condition, is the earthquake and being the basal shear, the lateral force applied in the building proportional to the weight of the structure, so that an alternative to mitigate this seismic action on reinforced concrete structures is to reduce the weight of structural elements by using lightweight concrete. Therefore, the main objective of the research is to manufacture a low-density concrete that in turn has structural characteristics and also can be determined by tests and correlations the physical and mechanical parameters of lightweight concrete so that they can be used as data within the analysis and structural design of buildings.

On the basis of what has been described, the investigation consists in the elaboration of a low-density structural concrete with the use of light aggregate (pumice stone) extracted from the San Antonio - Calacalí road section, fine aggregate from the Pifo quarry and GU type cement. general use. The structural lightweight concrete design is based on three standards: ACI 318-14: Code requirements for structural concrete, ACI 211.1-98 Standard practice for selecting proportions of lightweight structural concrete and ACI 213R-14: Guide for light aggregate structural concrete, which provide tables for estimating the quantities of material, depending on the resistance you want to achieve.

The dosage for one cubic meter of structural lightweight concrete is 9.2 bags of cement, 208 liters of water, $792 \mathrm{~kg}$ of sand, 326 $\mathrm{kg}$ of pumice stone and $4.6 \mathrm{~kg}$ of Aditec SF - 106 superplasticizer additive. Using this dosage, a concrete is obtained structural light with a 28-day compressive strength of $282 \mathrm{~kg} / \mathrm{cm} 2$ and equilibrium density of $1.81 \mathrm{~kg} / \mathrm{cm} 3$, in addition to be used later in a mathematical model, the modulus of rupture, tensile strength is obtained, modulus of elasticity, Poisson module through tests and correlation formulas of the ACI. Lightweight concrete can be used in structural elements such as beams, columns and slabs, but cannot be in direct contact with water due to its porosity. The decrease in the density of concrete brings many advantages such as reduction of loads, sections, amount of steel and even cost.
\end{abstract}

Keywords: lightweight structural concrete, pumice stone, low density concrete. 


\section{Introducción}

El hormigón liviano con el uso de áridos ligeros fue utilizado por los romanos en la construcción de la cúpula del Panteón de Agripa de 44 metros de diámetro, que data del año 118 y 125 d. C. [1].

Existen diversos estudios realizados en el Ecuador sobre el hormigón ligero; uno de ellos, por ejemplo, es el efectuado en la Universidad Central del Ecuador denominado:

"Análisis comparativo entre hormigón convencioal y hormigón de baja densidad", en el cual se consiguieron hormigones de densidad alrededor de los $1.88 \mathrm{~kg} / \mathrm{cm}^{3}$ y resistencias a la compresión a los 28 días de $180 \mathrm{~kg} / \mathrm{cm}^{2}$.

En esta investigación se comprobó que se pueden obtener hormigones con densidad baja $(1,81 \mathrm{~kg} /$ $\mathrm{cm}^{3}$ ), pero a la vez se planteó conseguir hormigones con resistencias mayores a los $180 \mathrm{~kg} / \mathrm{cm}^{2}$, de acuerdo a los ensayos se cumplió el propósito, obteniendo resistencias de hasta $280 \mathrm{~kg} / \mathrm{cm}^{2}$, que permite definir al hormigón como estructural y que este sea empleado en elementos estructurales como columnas, vigas y losas.

\section{Fundamentación}

El diseño de hormigón ligero estructural se basa principalmente en las siguientes normas:

- ACI 318-14: Requisitos del Código para hormigón estructural.

- ACI 211.1-98: Práctica estándar para seleccionar proporciones de hormigón estructural ligero.

- ACI 213R-14: Guía para concreto estructural agregado ligero.

\section{Definiciones}

Hormigón ligero estructural: hormigón que posee una resistencia a la compresión mínima a los 28 días de $17 \mathrm{MPa}$, una densidad de equilibrio entre 1120 y $1920 \mathrm{~kg} / \mathrm{m} 3$, y consiste completamente de agregado de peso ligero o una combinación de peso ligero y agregado de densidad normal. [2]

Densidad de equilibrio: es la densidad alcanzada por el hormigón estructural ligero después de la exposición a la humedad relativa de $50 \pm 5 \%$ y una temperatura de $23 \pm 2{ }^{\circ} \mathrm{C}$ por un período de tiempo suficiente para alcanzar una densidad que cambia menos del $0,5 \%$ en un período de 28 días. [2]

Agregado ligero: es aquel que tiene un peso volumétrico seco suelto como máximo $880 \mathrm{~kg} / \mathrm{m} 3$, mientras que para el agregado normal las densidades oscilan entre 14440 a $1769 \mathrm{~kg} / \mathrm{m} 3$. [3]

\section{Formulación de objetivos y establecimiento de hipótesis}

\subsection{Objetivo general}

- Fabricar un hormigón ligero estructural que pueda usarse como material para el análisis y diseño de edificaciones.

\subsection{Objetivos específicos}

- Determinar la resistencia a la compresión simple del hormigón ligero a los 7, 14 y 28 días.

- Obtener la densidad de equilibrio que defina al hormigón como ligero.

- Establecer el módulo de rotura.

- Definir la resistencia a la tracción.

- Calcular mediante fórmulas o correlaciones el módulo de elasticidad Ec del hormigón ligero.

\subsection{Hipótesis}

Es posible conseguir un hormigón de baja densidad que a su vez posea una resistencia estructural con el uso de piedra pómez. 


\section{Materiales, fuentes y métodos}

\subsection{Materiales}

El desarrollo del proyecto se centra en la provincia de Pichincha, donde se utilizará materiales locales. El hormigón ligero tiene varios componentes como son, el cemento Portland tipo IP, arena de peso normal, agua, aditivo y piedra pómez.

\section{Piedra pómez}

Piroclasto de caída con un tamaño comprendido entre 2 y $64 \mathrm{~mm}$, generado en erupciones explosivas a partir de la fragmentación de la lava que recubre las burbujas de gas que ascienden hacia la superficie y explotan por la diferencia de su presión interna con la del entorno. [4]

\section{Agregado fino}

El agregado fino consiste en arena natural proveniente de canteras aluviales o de arena producida artificialmente. La forma de las partículas es generalmente cúbica o esférica y razonablemente libre de partículas delgadas, planas o alargadas. La arena natural es constituida por fragmentos de roca limpios, duros, compactos, durables. [4]

\section{Cemento}

El cemento es un conglomerante hidráulico, es decir, un material inorgánico finamente molido que, amasado con agua, forma una pasta que fragua y endurece por medio de reacciones y procesos de hidratación y que, una vez endurecido conserva su resistencia y estabilidad incluso bajo el agua. [5]

\section{Agua}

El agua es un componente esencial en las mezclas de concreto y morteros, pues permite que el cemento desarrolle su capacidad ligante.

El agua utilizada en la elaboración del concreto y mortero debe ser apta para el consumo humano, libre de sustancias como aceites, ácidos, sustancias alcalinas y materias orgánicas. [6]

\section{Aditivo superplastificante}

Los aditivos plastificantes y superplastificantes de hormigón son aditivos para hormigón capaces de mejorar las propiedades del hormigón.

Se emplean para conferir al hormigón fresco un mejor comportamiento en cuanto a trabajabilidad y bombeabilidad, pero también se busca con su uso mejorar significativamente la resistencia y la durabilidad del hormigón final. [7]

\subsection{Fuentes}

La calidad del hormigón depende del tipo de agregado que se utilice en la construcción. En Quito operan 65 canteras que abarcan 1.739 hectáreas, distribuidas en las parroquias de San Antonio de Pichincha, Píntag, Calderón, Pifo, Lloa y Guayllabamba.

Según el Municipio, el 69\% corresponde a concesiones otorgadas a particulares, el $27 \%$ a autorizaciones de libre aprovechamiento para obra pública y $4 \%$ a permisos para minería artesanal. Además, hay aquellas que trabajan de manera ilegal. [8]

Buena parte de los materiales de construcción para la ciudad provienen del norte de Quito, la mayoría de los sitios de extracción de los agregados se ubican en el tramo de vía Quito-Mitad del Mundo - Perucho, en la provincia existen otras fuentes de materiales como Guayllabamba, Pifo, Calacalí, Lloa, Píntag, entre otras, de donde se puede obtener los agregados para la fabricación del hormigón.

Para el desarrollo de este estudio del hormigón ligero se utilizó los agregados provenientes de las de zonas de Pifo y Mitad del Mundo - Calacalí.

\subsection{Métodos}

\section{Muestra}

La piedra pómez fue extraída del tramo de la vía Mitad del Mundo-Calacalí, sector La Cruz, el agregado fino de peso normal de la cantera de Pifo, Cemento Holcim G.U. y el aditivo ADITEC SF-106 que se adquirió de la empresa Aditec Ecuatoriana Cía. Ltda. 


\section{Técnicas}

Se efectuó los ensayos de laboratorio a los agregados, basándose en las normas INEN y ASTM que justifiquen la calidad de los materiales que van a intervenir en el proceso de fabricación del hormigón ligero.

\section{Recopilación de datos}

\subsection{Ensayos de caracterización de los materiales}

Para la caracterización de los agregados se realizaron varios ensayos en el laboratorio que incluyen la determinación de la densidad aparente real, suelta y compactada, granulometría, absorción, y contenido de humedad.

Tabla 1. Datos de las propiedades de los agregados

\begin{tabular}{lccc}
\hline & Unidad & Arena & Pómez \\
\hline $\begin{array}{l}\text { Contenido de } \\
\text { humedad (CH) }\end{array}$ & $\%$ & 0.89 & 5.79 \\
\hline $\begin{array}{l}\text { Contenido de } \\
\text { absorción (CA) }\end{array}$ & $\%$ & 2.57 & 33.61 \\
\hline $\begin{array}{l}\text { Densidad en estado } \\
\text { SSS (DSSS) }\end{array}$ & $\left(\mathrm{gr} / \mathrm{cm}^{3}\right)$ & 2.58 & 1.23 \\
\hline $\begin{array}{l}\text { Densidad seca } \\
\text { compactada (DSC) }\end{array}$ & $\left(\mathrm{gr} / \mathrm{cm}^{3}\right)$ & 1.7 & 0.53 \\
\hline $\begin{array}{l}\text { Densidad seca suelta } \\
\text { (DSS) }\end{array}$ & $\left(\mathrm{gr} / \mathrm{cm}^{3}\right)$ & 1.57 & 0.47 \\
\hline $\begin{array}{l}\text { Módulo de finura } \\
\text { (MF) }\end{array}$ & --- & 3.02 & 2.78 \\
\hline $\begin{array}{l}\text { Tamaño nominal } \\
\text { máximo TNM }\end{array}$ & pulgada & & $3 / 8 \%$ \\
\hline
\end{tabular}

Fuente: Los autores

Peso específico del cemento Holcim G.U.

P.E. $=2.90 \mathrm{gr} / \mathrm{cm}^{3}$

\section{Diseño del hormigón estructural ligero}

El método se basa principalmente en la norma ACI 211.2-98 Standard Practice for Selecting Proportions for Structural Lightweight Concrete.

Este método de diseño es aplicable para mezclas hechas con agregado gruesos ligeros, agregados finos ligeros y agregado finos de peso normal, el cual proporciona la secuencia de pasos para poder hacer la dosificación del hormigón ligero. [9]
Proceso para el diseño de mezclas de hormigón ligero

1) Estudio de las especificaciones de la obra.

Dependiendo de la resistencia y densidad requeridas se procederá al diseño del hormigón ligero.

2) Definición de la resistencia promedio requerida

Cuando una instalación productora de concreto no tenga registros de ensayos de resistencia en obra para el cálculo de Ss que se ajuste a los requisitos de la desviación estándar, el f'cr debe determinarse de la tabla 2.

Tabla 2. Resistencia promedio a la compresión requerida cuando no hay datos disponibles para establecer una desviación estándar de la muestra.

\begin{tabular}{ll}
\hline $\begin{array}{l}\text { Resistencia } \\
\text { especificada a la } \\
\text { compresión, MPa }\end{array}$ & $\begin{array}{l}\text { Resistencia promedio } \\
\text { requerida a la compresión, } \\
\text { MPa }\end{array}$ \\
\hline $\mathrm{f}^{\prime} \mathrm{c}<21$ & $\mathrm{f}^{\prime} \mathrm{cr}=\mathrm{f}^{\prime} \mathrm{c}+7.0$ \\
\hline $21 \leq \mathrm{f}^{\prime} \mathrm{c} \leq 35$ & $\mathrm{f}^{\prime} \mathrm{cr}=\mathrm{f}^{\prime} \mathrm{c}+8.3$ \\
\hline $\mathrm{f}^{\prime} \mathrm{c}>35$ & $\mathrm{f}^{\prime} \mathrm{cr}=1.10 \mathrm{f}^{\prime} \mathrm{c}+5.0$ \\
\hline
\end{tabular}

Fuente: ACI, American Concrete Institute. Requisito de Reglamento para Concreto Estructural ACI 318S-08. Impreso en U.S.A. Año 2008. Pág.: 72.

\section{3) Estimación del revenimiento}

Elección del revenimiento si no se especifica, el ACI proporciona una tabla de revenimiento con valores recomendados para cada tipo de elementos estructurales que se desea construir.

Tabla 3. Revenimiento recomendado para diferentes elementos estructurales

\begin{tabular}{lll}
\hline & \multicolumn{2}{l}{ Revenimiento(mm) } \\
\hline Elemento estructural & Máximo & Mínimo \\
\hline Vigas y muros reforzados & 100 & 25 \\
\hline Columnas & 100 & 25 \\
\hline Sistemas de piso (losas) & 75 & 25 \\
\hline
\end{tabular}

Fuente: ACI, American Concrete Institute. Standard Practice for Selecting Proportions for Structural Lightweight Concrete (ACI 211.2-98). Impreso en U.S.A. Año 2004. Pág.: 211.2-5. 
4) Estimación del agua de la mezcla y el contenido de aire

La cantidad de agua por unidad de volumen para obtener el revenimiento deseado depende del tamaño máximo, de la forma y de la graduación del agregado, del contenido de aire y de los aditivos químicos que se empleen, no depende en gran medida de la cantidad del cemento. La tabla 4 proporciona una estimación del agua requerida dependiendo del tamaño máximo de agregado y la cantidad de aire. La forma y la textura de los agregados influye en la cantidad de agua, pero los valores de la tabla dan una suficiente aproximación para esta primera estimación.

Tabla 4. Requerimientos aproximados de agua de mezcla para diferentes revenimientos y tamaño máximo de agregado

\begin{tabular}{|c|c|c|c|}
\hline \multirow[t]{2}{*}{ Tamaño máximo de agregado } & $3 / 8 ”$ & $1 / 2 "$ & $3 / 4 "$ \\
\hline & $(9.5 \mathrm{~mm})$ & $(12.7 \mathrm{~mm})$ & $(19 \mathrm{~mm})$ \\
\hline \multicolumn{4}{|l|}{ Concreto con aire incluido } \\
\hline & \multicolumn{3}{|c|}{ Agua en $\mathrm{Kg} / \mathrm{m}^{3}$ de concreto } \\
\hline Revenimiento de $25 \mathrm{~mm}$ a $50 \mathrm{~mm}$ & 181 & 175 & 166 \\
\hline Revenimiento de $75 \mathrm{~mm}$ a $100 \mathrm{~mm}$ & 202 & 193 & 181 \\
\hline \multirow[t]{2}{*}{ Revenimiento de $125 \mathrm{~mm}$ a $150 \mathrm{~mm}$} & 211 & 199 & 187 \\
\hline & \multicolumn{3}{|c|}{$\begin{array}{l}\text { Contenido total de aire recomendado para diferentes ni- } \\
\text { veles de exposiciones, } \%\end{array}$} \\
\hline Exposición ligera & 4.5 & 4 & 4 \\
\hline Exposición moderada & 6 & 5.5 & 5 \\
\hline Exposición extrema & 7.5 & 7 & 6 \\
\hline \multicolumn{4}{|l|}{ Concreto sin aire incluido } \\
\hline & \multicolumn{3}{|c|}{ Agua en $\mathrm{kg} / \mathrm{m}^{3}$ de concreto } \\
\hline Revenimiento de $25 \mathrm{~mm}$ a $50 \mathrm{~mm}$ & 208 & 199 & 187 \\
\hline Revenimiento de $75 \mathrm{~mm}$ a $100 \mathrm{~mm}$ & 228 & 217 & 202 \\
\hline \multirow[t]{3}{*}{ Revenimiento de $125 \mathrm{~mm}$ a $150 \mathrm{~mm}$} & 237 & 222 & 208 \\
\hline & \multicolumn{3}{|c|}{$\begin{array}{l}\text { Contenido aproximado de aire atrapado en el concreto } \\
\text { sin aire incluido en } \%\end{array}$} \\
\hline & 3 & 2.5 & 2 \\
\hline
\end{tabular}

Fuente: ACI, American Concrete Institute. Standard Practice for Selecting Proportions for Structural Lightweight Concrete (ACI 211.2-98), Impreso en U.S.A. Año 2004. Pág.: 211.2-5.

En la tabla se indica los niveles recomendados de contenido de aire con respecto a la exposición que tendrá el concreto en servicio. Estos valores mejoran el manejo y la durabilidad de la mezcla, ayudando a reducir la masa de esta.

Según el ACI 213R-03, Capítulo 3, Tabla 3.1 se tiene valores de contenido de aire recomendado para el concreto ligero para diferentes tamaños máximos nominales.
Tabla 5. Contenido de aire recomendado para el concreto ligero.

\begin{tabular}{|c|c|}
\hline $\begin{array}{l}\text { Tamaño máximo de } \\
\text { agregado }\end{array}$ & $\begin{array}{l}\text { Contenido de aire } \\
\text { por } \% \text { en volumen }\end{array}$ \\
\hline $3 / 4 "$ pulgada $(19 \mathrm{~mm})$ & 4.5 a 7.5 \\
\hline $3 / 8 "$ pulgada $(10 \mathrm{~mm})$ & 6 a 9 \\
\hline
\end{tabular}


5) Selección de la relación aproximada agua cemento

En la relación agua-cemento w/c a emplear, no solo interviene la resistencia requerida sino también factores como la durabilidad y las propiedades finales deseadas. Dado que cuando se utilizan diferentes agregados y cementantes con la misma relación agua cemento resultan diferentes resistencias, es deseable desarrollar una relación entre la resistencia y w/c para cada material que se utilice.

Tabla 6. Relación entre w/c y resistencia a compresión del concreto.

\begin{tabular}{|c|c|c|}
\hline \multirow{2}{*}{$\begin{array}{l}\text { Resistencia a } \\
\text { compresión a } \\
28 \text { días } \mathrm{MPa}\end{array}$} & \multicolumn{2}{|c|}{$\begin{array}{l}\text { Relación aproximada agua } \\
\text { cemento, por masa }\end{array}$} \\
\hline & $\begin{array}{l}\text { Concreto sin } \\
\text { aire incluido }\end{array}$ & $\begin{array}{l}\text { Concreto con } \\
\text { aire incluido }\end{array}$ \\
\hline 41.4 & 0.41 & ---- \\
\hline 34.5 & 0.48 & 0.4 \\
\hline 27.6 & 0.57 & 0.48 \\
\hline 20.7 & 0.68 & 0.59 \\
\hline 13.8 & 0.82 & 0.74 \\
\hline
\end{tabular}

Fuente: ACI, American Concrete Institute. Standard Practice for Selecting Proportions for Structural Lightweight Concrete (ACI 211.2-98). Impreso en U.S.A. Año 2004. Pág.: 211.2-6.

\section{6) Cálculo de la cantidad de cemento}

Una vez que la cantidad de agua y la relación w/c han sido estimados, se puede obtener fácilmente la cantidad de cemento por unidad de volumen del concreto que es determinada dividiendo la cantidad de agua por la relación w/c.

Cemento $\left(\frac{\mathrm{kg}}{\mathrm{m}^{3}}\right)=\frac{\text { Cantidad de agua }}{\frac{w}{c}\left(\text { para } f^{\prime} c r\right)}$

\section{Ecuación N. 1}

7) Determinación del contenido del agregado grueso ligero

Agregados con el mismo tamaño máximo nominal y graduación producen concretos con una manejabilidad satisfactoria con un apropiado volumen de agregado grueso, en la tabla 7 se proporciona una estimación de dicho volumen, este valor depende solamente del tamaño máximo del agregado y módulo de finura del agregado fino.

Tabla 7. Volumen de agregado grueso por unidad de volumen de concreto.

\begin{tabular}{llllll}
\hline $\begin{array}{l}\text { T a m a } \\
\text { máximo o } \\
\text { agregado }(\mathrm{mm})\end{array}$ & $\begin{array}{l}\text { Volumen del agregado grueso } \\
\text { suelto en horno por unidad } \\
\text { de volumen de concreto para } \\
\text { diferentes módulos de finura de } \\
\text { arena }\end{array}$ \\
\hline pulgada & $\mathrm{mm}$ & 2.4 & 2.6 & 2.8 & 3 \\
\hline $3 / 8$ & 9.5 & 0.58 & 0.56 & 0.54 & 0.52 \\
\hline $1 / 2$ & 12.7 & 0.67 & 0.65 & 0.63 & 0.61 \\
\hline $3 / 4$ & 19 & 0.74 & 0.72 & 0.7 & 0.68 \\
\hline
\end{tabular}

Fuente: ACI, American Concrete Institute. Standard Practice for Selecting Proportions for Structural Lightweight Concrete (ACI 211.2-98). Impreso en U.S.A. Año 2004. Pág.: 211.2-6.

8) Determinación del contenido del agregado fino

A la finalización del paso 7, se han estimado todos los ingredientes del concreto excepto el agregado fino. A la determinación de la cantidad de arena se puede llegar por dos caminos que resultan al final en establecer el valor por diferencia.

El primer camino se refiere a que en ocasiones por experiencias en este tipo de mezclas se puede conocer la masa volumétrica con suficiente aproximación y una vez obtenido este valor, se determina la masa por diferencia, en ausencia de dichas experiencias, se puede recurrir a la tabla 8 que proporciona una primera estimación basada en el factor de gravedad específica del agregado grueso y el contenido de aire. 
Tabla 8. Primera estimación de la masa volumétrica del concreto ligero fresco compuesto por agregado grueso ligero y arena de masa normal.

\begin{tabular}{llll}
\hline $\begin{array}{l}\text { Factor de } \\
\text { gravedad } \\
\text { específica }\end{array}$ & $\begin{array}{l}\text { Primera estimación de la masa } \\
\text { volumétrica del concreto ligero, } \\
\mathbf{k g} / \mathbf{m}^{\mathbf{3}}\end{array}$ \\
\cline { 2 - 4 } & \begin{tabular}{l} 
Concreto con aire incluido \\
\cline { 2 - 4 }
\end{tabular} & $\begin{array}{l}\text { 4\% } \\
\text { (15) }\end{array}$ \\
\hline $\mathbf{1}$ & 1596 & 1561 & 1519 \\
\hline $\mathbf{1 . 2}$ & 1680 & 1644 & 1608 \\
\hline $\mathbf{1 . 4}$ & 1769 & 1727 & 1691 \\
\hline $\mathbf{1 . 6}$ & 1852 & 1810 & 1775 \\
\hline $\mathbf{1 . 8}$ & 1935 & 1899 & 1858 \\
\hline $\mathbf{2}$ & 2024 & 1982 & 1941 \\
\hline
\end{tabular}

Fuente: ACI, American Concrete Institute. Standard Practice for Selecting Proportions for Structural Lightweight Concrete (ACI 211.2-98). Impreso en U.S.A. Año 2004. Pág.: 211.2-7.

\section{9) Ajustes en las proporciones de la mezcla}

Consiste en ajustar las mezclas por humedad y absorción a los agregados, la cantidad de agua que aportarán o absorberán los agregados, según esto se realiza una corrección al valor total de agua, de esta manera se tiene una primera estimación de las cantidades a emplear en la mezcla de concretos ligeros.

\section{Dosificación del hormigón ligero estructural f'c $280 \mathrm{~kg} / \mathrm{cm}^{2}$.}

Tabla 9. Cantidades por metro cúbico de hormigón ligero estructural

\begin{tabular}{lll}
\hline Material & Pesos kg & Dosificación \\
\hline Agua & 208 & 0.45 \\
\hline Cemento & 462.22 & 1 \\
\hline Arena & 792.40 & 1.71 \\
\hline Pómez & 326.54 & 0.71 \\
\hline Aditivo & 4.62 & 0.01 \\
\hline
\end{tabular}

Fuente: Los autores
Tabla 10. Cantidades para 12 cilindros $\emptyset=10 \mathrm{~cm}, \mathrm{~h}=20 \mathrm{~cm}$.

\begin{tabular}{lll}
\hline Material & Pesos kg & Dosificación \\
\hline Agua & 6.820 & 0.44 \\
\hline Cemento & 15.501 & 1.00 \\
\hline Arena & 26.573 & 1.71 \\
\hline Pómez & 10.951 & 0.71 \\
\hline Aditivo & 0.1550 & 0.01 \\
\hline \multicolumn{3}{l}{ Fuente: Los autores }
\end{tabular}

\section{Proceso de fabricación del hormigón ligero}

Para la fabricación del hormigón ligero se debe seguir algunos pasos en el mezclado:

1. La piedra pómez debe cumplir con los requisitos de tamaño y granulometría descritos en la norma ASTM C330: Especificación estándar para agregados livianos para concreto estructural.

2. Antes de iniciar con el proceso de fabricación del hormigón estructural ligero al agregado liviano (piedra pómez) se le sumerge en el agua aproximadamente 24 horas, es preferible usar a la piedra pómez en estado saturado en superficie seca (S.S.S.), debido a que tiende a absorber menos agua y no se vea afectada el agua de diseño de la mezcla.

3. Se procede a pesar los materiales calculados para la mezcla, el proceso de mezclado se realizó mediante la concretera a la cual previamente se humedeció antes de colocar los componentes del hormigón ligero.

4. En las mezcladoras de eje vertical es importante primero colocar en el tambor el agregado grueso ligero (piedra pómez) y seguidamente con la concretera en marcha se añade las $2 / 3$ parte del agua de diseño para la pre-saturación, una forma de guiarse es que cuando el material está en ese estado las partículas no se pegan a las paredes de la concretera, al agregado ligero se lo deja mezclar por unos 50 segundos aproximadamente.

5. Con mucho cuidado se hace girar el tambor hasta una posición que permita colocar el 
agregado fino (arena), cemento y colocar las $3 / 4$ partes del agua de diseño.

6. Dejar mezclar por unos 2 minutos, se considera que a mayor tiempo de mezclado influye beneficiosamente en la docilidad del hormigón fresco con este tipo de agregado.

7. Se procede a añadir el aditivo con el agua restante de la mezcla y extender el tiempo de mezclado mínimo de 3 minutos hasta obtener una mezcla fluida y proceder a la descarga en una bandeja grande.

8. Debido a la baja densidad que presenta el agregado la compactación del hormigón varía, ya que el comportamiento del árido en el hormigón difiere del árido de densidad normal. Puesto que los áridos livianos tienden a amortiguar fuertemente las vibraciones, mientras que los áridos de peso normal se compactan al poco tiempo. Pasa lo mismo el momento de expulsar el aire contenido después de la compactación, ya que la movilidad de los áridos de peso normal en el hormigón resulta más favorable que para agregados de baja densidad, por lo cual, el número de inmersiones de la varilla y el número de golpes con el mazo se estima al doble. A continuación, se presenta la tabla 11, que resume los parámetros adoptados para la mezcla.
Tabla 11. Pautas para la elaboración de cilindros de hormigón ligero

\begin{tabular}{lc}
\hline Mezcla & HLE \\
\hline Pre-saturación del agregado (seg) & 50 \\
\hline Tiempo total de mezcla (seg) & 6 \\
\hline Número de penetraciones de varilla & 30 \\
\hline Número de golpes de martillo de caucho & 25 \\
\hline
\end{tabular}

Fuente: (Caiza \& Martínez, 2015, págs. 21-40)

\section{Tratamiento de las variables}

La determinación de las propiedades en estado fresco se realizó mediante varios ensayos, entre los que destacan la consistencia que se determinó mediante el ensayo de cono de Abrams ASTM C143, densidad fresca ASTM C138, y homogeneidad.

A los 28 días se midió las propiedades mecánicas más importantes entre las principales resistencias a la compresión ASTM C39, módulo de elasticidad ASTM C469, densidad seca ASTM C567, ensayo de tracción indirecta ASTM C496 y módulo de rotura ASTM C78.

\section{Resultados}

Se realizó el control de calidad del hormigón, en estado fresco se tomó densidad y asentamiento, en estado endurecido las principales propiedades mecánicas tomadas a los 28 días de fabricación y la evolución de la resistencia a los 7, 14 y 28 días respectivamente.

Tabla 12. Valores de asentamientos del hormigón ligero

\begin{tabular}{cccc}
\hline \multirow{2}{*}{ Canteras } & \multirow{2}{*}{ Métodos } & Asentamiento & Consistencia \\
\cline { 3 - 4 } & & $\mathbf{c m}$ & Tipos \\
\hline \multirow{2}{*}{ Pifo } & & 8.5 & Blanda \\
\cline { 3 - 4 } Mitad del Mundo-Calacalí & \multirow{2}{*}{ ASTM } & 9 & Blanda \\
\cline { 3 - 4 } & & 8.5 & Blanda \\
\hline
\end{tabular}

Fuente: Los autores 
Tabla 13. Valores de densidad fresca del hormigón ligero

\begin{tabular}{ccccc}
\hline & Unidad & Ensayo 1 & Ensayo 2 & Ensayo 3 \\
\hline Volumen: & $\mathrm{g}$ & 2928 & 2928 & 2928 \\
\hline Peso del molde: & $\mathrm{g}$ & 1940 & 1940 & 1940 \\
\hline Peso del molde + muestra: & $\mathrm{g}$ & 7655 & 7649 & 7651 \\
\hline Peso de la muestra: & $\mathrm{g}$ & 5715 & 5709 & 5711 \\
\hline Densidad de la muestra: & $\mathrm{g} / \mathrm{cm} 3$ & 1.952 & 1.950 & 1.950 \\
\hline Densidad fresca promedio & $\mathrm{g} / \mathrm{cm} 3$ & 1.951 & & \\
\hline
\end{tabular}

Fuente: Los autores

Tabla 14. Valores de densidad endurecida del hormigón ligero

\begin{tabular}{|c|c|c|c|c|c|c|}
\hline N. & $\varphi \mathrm{m}$ & $\mathrm{hm}$ & $\mathrm{V}$ & Peso cilindro & $\gamma$ horm. & $\gamma$ horm promedio \\
\hline & $\mathrm{cm}$ & $\mathrm{cm}$ & $\mathrm{cm}^{3}$ & $g$ & $\mathrm{~g} / \mathrm{cm} 3$ & $\mathrm{~g} / \mathrm{cm} 3$ \\
\hline \multicolumn{7}{|c|}{7 días } \\
\hline 1 & 10.30 & 20.00 & 1666.4 & 3174 & 1.905 & \multirow{3}{*}{1.911} \\
\hline 2 & 10.27 & 20.10 & 1663.9 & 3208 & 1.928 & \\
\hline 3 & 10.33 & 20.00 & 1677.2 & 3187 & 1.900 & \\
\hline \multicolumn{7}{|c|}{14 días } \\
\hline 4 & 10.23 & 20.00 & 1644.9 & 3123 & 1.899 & \multirow{3}{*}{1.901} \\
\hline 5 & 10.27 & 20.03 & 1658.4 & 3158 & 1.904 & \\
\hline 6 & 10.30 & 20.03 & 1669.2 & 3172 & 1.900 & \\
\hline \multicolumn{7}{|c|}{28 días } \\
\hline 7 & 10.57 & 20.10 & 1762.6 & 3376 & 1.915 & \multirow{3}{*}{1.919} \\
\hline 8 & 10.43 & 19.97 & 1707.0 & 3286 & 1.925 & \\
\hline 9 & 10.30 & 20.00 & 1666.4 & 3195 & 1.917 & \\
\hline
\end{tabular}

Fuente: Los autores

Tabla 15. Valores de resistencia a compresión del hormigón ligero

\begin{tabular}{|c|c|c|c|c|c|}
\hline N. & $\varphi \mathrm{m}$ & A & Carga & $\sigma$ & $\sigma$ prom \\
\hline & $\mathrm{cm}$ & $\mathrm{cm}^{2}$ & $\mathrm{~kg}$ & $\mathrm{~kg} / \mathrm{cm}^{2}$ & $\mathrm{~kg} / \mathrm{cm}^{2}$ \\
\hline \multicolumn{6}{|c|}{7 días } \\
\hline 1 & 10.30 & 83.32 & 18350.00 & 220.23 & \multirow{3}{*}{222.06} \\
\hline 2 & 10.27 & 82.78 & 19120.00 & 230.96 & \\
\hline 3 & 10.33 & 83.86 & 18030.00 & 214.99 & \\
\hline \multicolumn{6}{|c|}{14 días } \\
\hline 4 & 10.23 & 82.25 & 21390.00 & 260.07 & \multirow{3}{*}{257.09} \\
\hline 5 & 10.27 & 82.78 & 20730.00 & 250.41 & \\
\hline 6 & 10.30 & 83.32 & 21730.00 & 260.79 & \\
\hline \multicolumn{6}{|c|}{28 días } \\
\hline 7 & 10.57 & 87.69 & 25718.00 & 293.27 & \multirow{3}{*}{282.30} \\
\hline 8 & 10.43 & 85.49 & 24203.00 & 283.10 & \\
\hline 9 & 10.30 & 83.32 & 22542.00 & 270.54 & \\
\hline
\end{tabular}

Fuente: Los autores 
Tabla 16. Valores de resistencia a la tracción indirecta del hormigón ligero

\begin{tabular}{|c|c|c|c|c|c|c|}
\hline $\mathbf{N}$ & $\varphi \mathbf{m}$ & Lm & Carga & $\sigma t$ & ot prom. & ot prom. \\
\hline & $\mathrm{cm}$ & $\mathrm{cm}$ & kg & $\mathrm{kg} / \mathrm{cm}^{2}$ & $\mathrm{~kg} / \mathrm{cm}^{2}$ & $\%$ \\
\hline 10 & 10.47 & 20.00 & 13173.00 & 40.06 & \multirow{3}{*}{35.66} & \multirow{3}{*}{12.63} \\
\hline 11 & 10.40 & 20.03 & 11929.00 & 36.45 & & \\
\hline 12 & 10.40 & 20.03 & 9974.00 & 30.48 & & \\
\hline
\end{tabular}

Fuente: Los autores

Tabla 17. Valores de módulo de rotura del hormigón ligero

\begin{tabular}{ccccccc}
\hline \multirow{2}{*}{ N. } & $\mathbf{b}$ & $\mathbf{d}$ & $\mathbf{L}$ & Carga & MR & MR prom. \\
\cline { 2 - 6 } & $\mathbf{c m}$ & $\mathbf{c m}$ & $\mathbf{c m}$ & $\mathbf{~ k g}$ & $\mathbf{k g} / \mathbf{c m}^{\mathbf{2}}$ & $\mathbf{k g} / \mathbf{c m}^{\mathbf{2}}$ \\
\hline V1 & 15.10 & 14.90 & 49.90 & 2280.00 & 33.94 & \\
\hline V2 & 15.00 & 15.10 & 50.00 & 2247.00 & 32.85 & \\
\hline V3 & 15.20 & 15.10 & 50.00 & 2055.00 & 29.65 & 32.14 \\
\hline
\end{tabular}

Fuente: Los autores

Tabla 18. Valores de módulo de elasticidad del hormigón ligero

\begin{tabular}{cccccc}
\hline \multirow{2}{*}{ Cilindro } & Densidad & \multicolumn{2}{c}{ Resistencia } & \multicolumn{2}{c}{ Módulo de elasticidad } \\
\cline { 2 - 6 } & $\mathrm{kg} / \mathrm{m} 3$ & $\mathrm{~kg} / \mathrm{cm} 2$ & $\mathrm{MPa}$ & $\mathrm{MPa}$ & $\mathrm{kg} / \mathrm{cm} 2$ \\
\hline 7 & 1915.3 & 293.2 & 28.7 & 19327 & 197135 \\
\hline 8 & 1924.9 & 283.1 & 27.7 & 19132 & 195152 \\
\hline 9 & 1917.2 & 270.5 & 26.5 & 18590 & 189625 \\
\hline
\end{tabular}

Fuente: Los autores

Los valores del módulo de elasticidad fueron calculados con la fórmula de correlación indicada en el ACI 318-14 para hormigones de densidad entre 1500 a $2500 \mathrm{~kg} / \mathrm{m}^{3}$.

$$
E_{c}=w_{c}{ }^{1.5} 0.043 \sqrt{f^{\prime} c} \text { en (MPa) Ecuación N. } 2
$$

Siendo $\mathrm{w}_{\mathrm{c}}$ la densidad del hormigón en $\mathrm{kg} / \mathrm{m}^{3} \mathrm{y} \mathrm{f}^{\prime} \mathrm{c}$ la resistencia promedio a la compresión a los 28 días en MPa.

\section{Módulo de Poisson}

Si bien esta propiedad varía ligeramente con la edad, las condiciones de prueba, y propiedades fí- sicas del concreto, un valor de 0.20 usualmente se asume para propósitos prácticos de diseño. [11]

Tabla 19. Datos de resistencia en diferentes edades

\begin{tabular}{lll}
\hline Tiempo & Resistencia & $\begin{array}{l}\text { Evolución de } \\
\text { resistencia }\end{array}$ \\
\hline Días & $\mathbf{k g} / \mathbf{c m} \mathbf{2}$ & $\%$ \\
\hline $\mathbf{0}$ & 0 & 0 \\
\hline $\mathbf{7}$ & 222.1 & 79.3 \\
\hline $\mathbf{1 4}$ & 257.1 & 91.8 \\
\hline $\mathbf{2 8}$ & 282.3 & 100.7 \\
\hline \multicolumn{3}{c}{ Fuente: Los autores }
\end{tabular}


Grafico 1. Resistencia a la compresión vs. Tiempo de hormigón ligero celular.

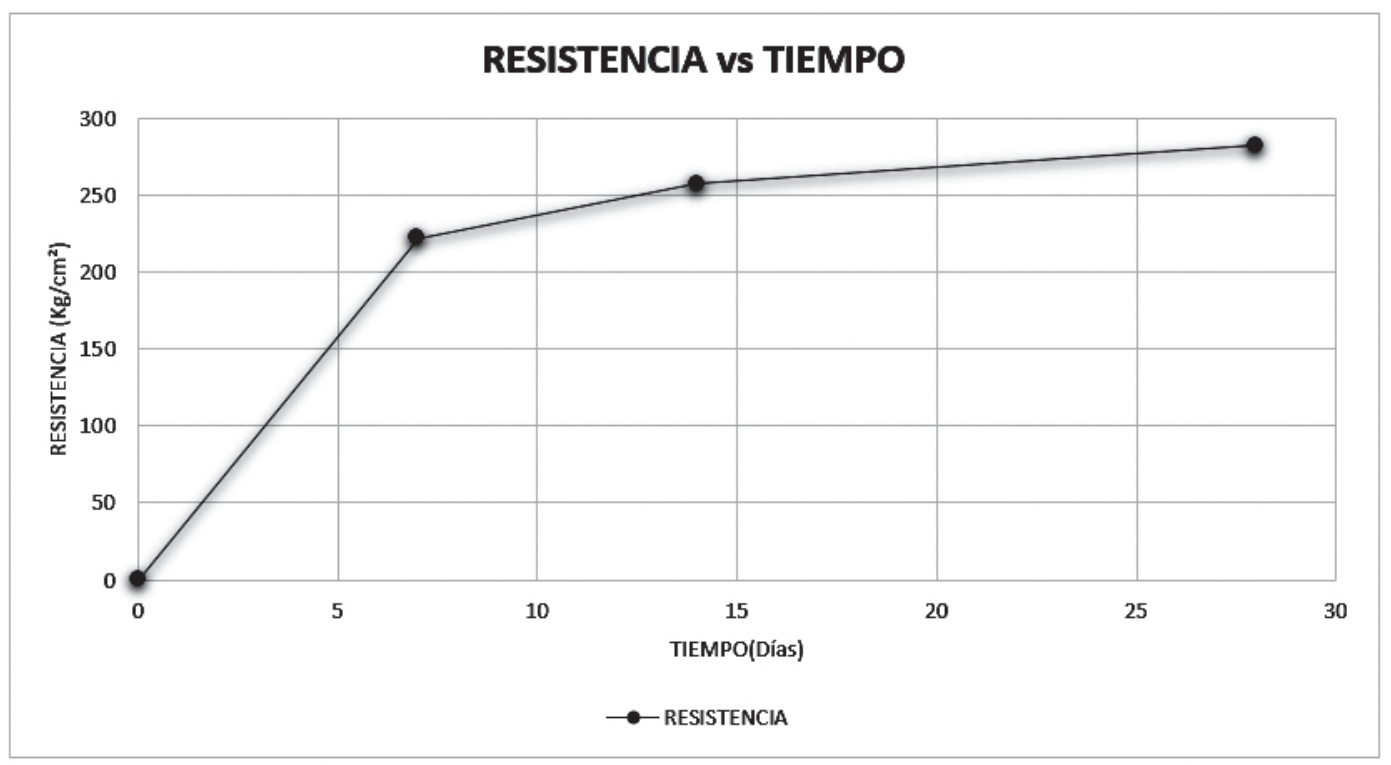

Fuente: Los autores

\section{Densidad aparente seca}

Las densidades que han sido monitoreadas son en estado endurecido a los 7, 14 y 28 días.

Tabla 20. Densidad del hormigón ligero a diferentes edades

\begin{tabular}{ll}
\hline Tiempo & Densidad \\
\hline Días & $\mathbf{k g} / \mathbf{m} \mathbf{3}$ \\
\hline $\mathbf{0}$ & 0 \\
\hline $\mathbf{7}$ & 1911 \\
\hline $\mathbf{1 4}$ & 1901 \\
\hline $\mathbf{2 8}$ & 1919 \\
\hline & Fuente: Los autores
\end{tabular}

Las mezclas para hormigones ligeros presentaron densidades entre $1901 \mathrm{~kg} / \mathrm{m}^{3}$ a $1919 \mathrm{~kg} / \mathrm{m}^{3}$, dentro del rango estipulado por el ACI 213R-03, y resultaron ser $13.77 \%$ más livianos que la misma mezcla combinada con piedra pómez y árido fino de peso normal.

\section{Conclusiones}

1) Con la combinación de agregado grueso liviano y agregado fino de peso normal, se llegó a obtener hormigones ligeros, con buenas ca- racterísticas físicas y mecánicas, cuyos datos a futuro servirán para disponer de un material ligero y con ello realizar un modelo matemático aplicando a un proyecto estructural real de una edificación.

2) Debido al alto porcentaje de absorción de la piedra pómez, las densidades obtenidas a los pocos días de haber retirado de la cámara de curado son mayores a la densidad de equilibrio cuyos valores están en el rango de $1810 \mathrm{a}$ $1830 \mathrm{~kg} / \mathrm{m} 3$, y además cumplen con la especificación de densidad del ACI 318S-14.

3) El uso de la piedra pómez como agregado grueso tiende a disminuir la resistencia a la compresión del hormigón, por ello se vio necesario aumentar la cantidad de cemento, tener una relación agua cemento más baja, y la utilización de aditivo superplasticante para compensar esta disminución en la resistencia y que a su vez sea trabajable.

4) Se obtuvieron valores de las propiedades físicas y mecánicas del hormigón ligero estructural apropiadas (ver tablas de la 12 a la 20), cuyos datos al compararse con valores de un hormigón convencional, cumplen los requerimientos técnicos y mecánicos para utilizarse 
dentro del análisis y diseño de elementos estructurales como columnas, vigas y losas en el caso de realizar.

\section{Referencias}

1) "Lightweight Concrete-A Proven Material for Two Millennia," Proceedings of Advances in Cement and Concrete, S. Sarkar and M. W. Grutzeck, eds., University of New Hampshire, Durham, S.C.

2) ACI 213-03R Guide for Structural Lightweight-Aggregate Concrete

3) ASTM C330 Especificación Normalizada para Agregados Livianos para Concreto Estructural

4) Konstruir.com. (2013). DICCIONARIO: Definición de agregado fino. Recuperado de: http://konstruir.com/definicion/letra_a/palabra_agregado\%20fino.html

5) IECA. (s. f.). Componentes y propiedades del cemento | Características - IECA. Recuperado de: https://www.ieca.es/componentes-y-propiedades-del-cemento/

6) Arias A., V. (2009). El agua del concreto. Elconcreto.blogspot.com. Recuperado de: http://elconcreto.blogspot.com/2009/01/elagua-del-concreto.html.

7) EHE-08. Instrucción española de hormigón estructural

8) ACI, American Concrete Institute. Standard Practice for Selecting Proportions for Structural Lightweight Concrete (ACI 211.2-98). Impreso en U. S.A. Año 2004.

9) ACI, American Concrete Institute. Requisito de Reglamento para Concreto Estructural ACI 318S-08. Impreso en U. S.A. Año 2008.

10) ACI, American Concrete Institute. Guide for Structural Lightweight-Aggregate Concrete (ACI 213R-03). Impreso en U. S.A. Año 2003.

11) Caiza, P., \& Martínez, A. (2015). Fabricación de hormigones livianos con materiales volcanoclásticos (lapilli) y su influencia en la reducción de fuerzas sísmicas.

\section{Elementos gráficos}

Figura 1. Extracción del agregado grueso, vía Mitad del Mundo-Calacalí.

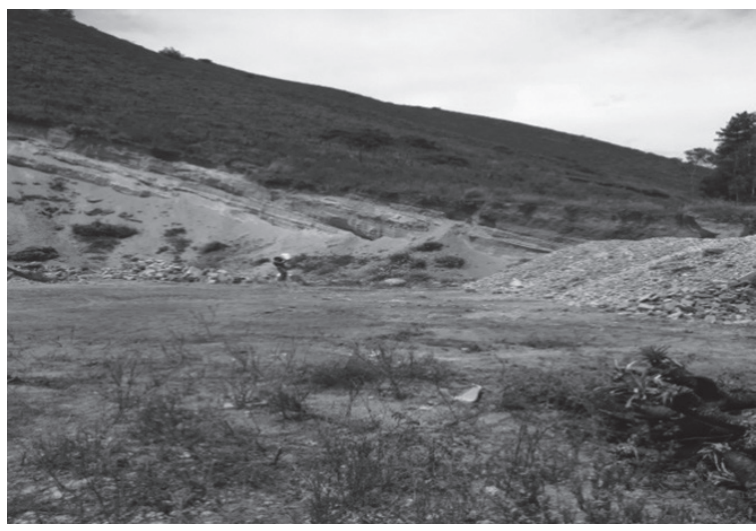

Fuente: Los autores

Figura 2. Extracción del agregado fino de la cantera Pifo.

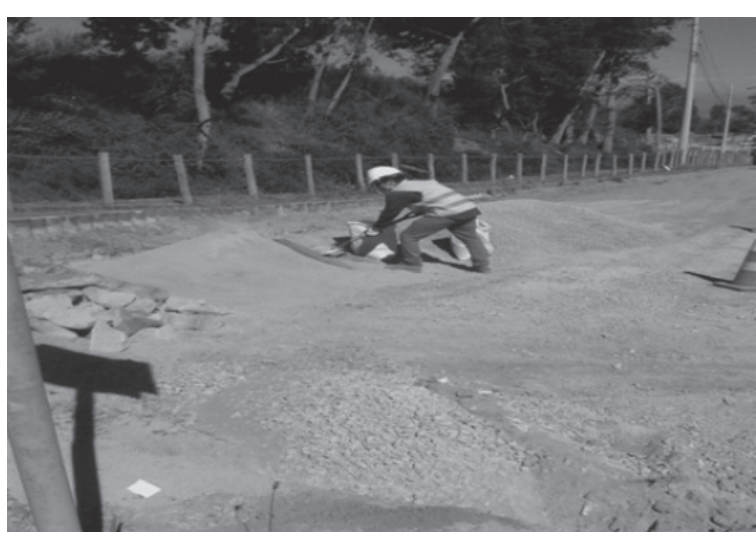

Fuente: Los autores

Figura 3. Fabricación del hormigón ligero.

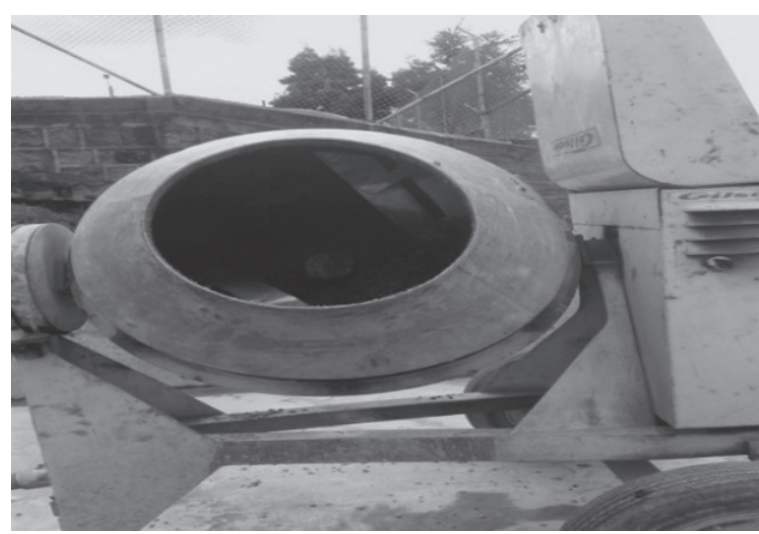

Fuente: Los autores 
Figura 4. Curado del hormigón.

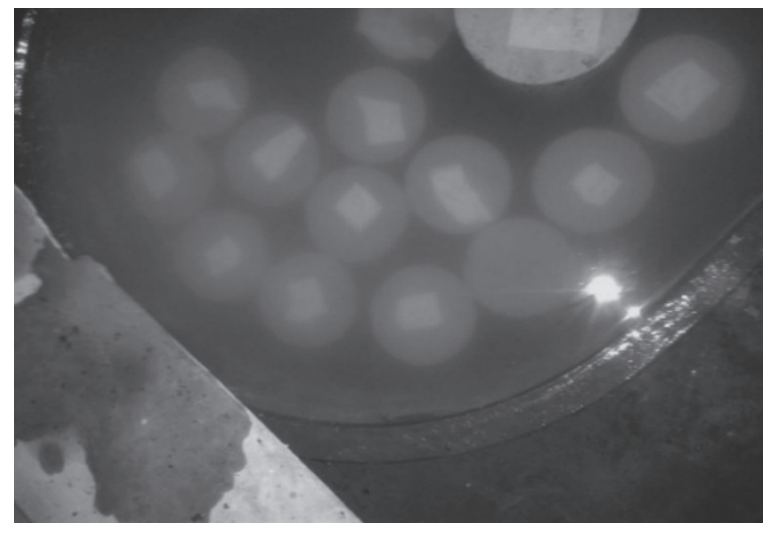

Fuente: Los autores

Figura 5. Ensayo a compresión.

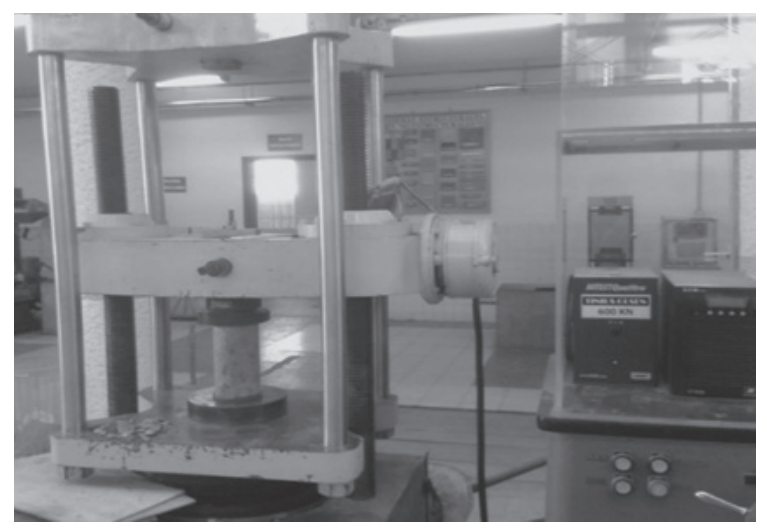

Fuente: Los autores

Figura 6. Ensayo a flexión

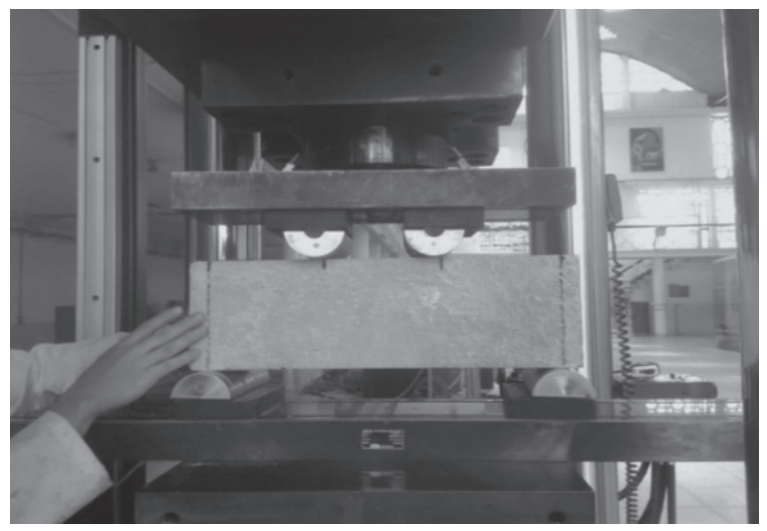

Fuente: Los autores.

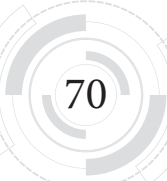

